\title{
Il Rapporto nazionale 2004 sull'uso dei farmaci in Italia
}

\author{
Mario Eandi
}

Pochi giorni prima della chiusura di questo numero è stato presentato il Rapporto Nazionale 2004 sull'uso dei farmaci in Italia, curato dall' OsMED (Osservatorio Nazionale sull'impiego dei Medicinali, organo dell'Agenzia Italiana del Farmaco) e pubblicato dal Ministero della Salute (giugno 2005). Integrazioni ed approfondimenti del rapporto sono disponibili in formato elettronico all'indirizzo Internet:

http://www.agenziafarmaco.it.

Lo studio è stato organizzato in tre parti, secondo una continuità di impostazione editoriale rispetto alle precedenti edizioni del rapporto.

Nella prima parte (A), dopo aver documentato i metodi di analisi, le fonti dei dati e i criteri di classificazione adottati, vengono descritte le caratteristiche generali della prescrizione di farmaci in Italia, evidenziando le correlazioni con età e sesso della popolazione, la dinamica temporale e le diagnosi fatte dai medici di Medicina Generale. I consumi dei farmaci nel periodo 2000-2004 vengono poi analizzati per singola classe terapeutica e commentati in relazione alla variabilità regionale, alla disponibilità di prodotti “generici” e alla classificazione per fasce di rimborsabilità. Le analisi si caratterizzano per l'adozione di possibili chiavi di lettura circa l'appropriatezza d'uso dei farmaci e per il tentativo di evidenziare l'impatto sulla dinamica della spesa farmaceutica dei provvedimenti adottati nel 2004 a livello nazionale e regionale.

La seconda (B) e terza (C) parte sono costituite da un insieme di tabelle e figure che illustrano i consumi e la spesa farmaceutica (rispettivamente a livello nazionale e a livello regionale), a diversi livelli di aggregazione dei farmaci, dalle classi terapeutiche fino alla singola molecola.

Tre Appendici, dedicate rispettivamente al complesso delle norme che regolano l'assistenza farmaceutica in Italia, alla classificazione (per molecola) adottata nelle analisi dell'effetto mix e alle pubblicazioni del 2004 relative a studi di farmacoepidemiologia e farmacoutilizzazione condotti in Italia, completano il rapporto.

La peculiarità di questo rapporto consiste nella base dei dati utilizzati per le elaborazioni e le valutazioni: si tratta di dati che derivano dall'integrazione dei flussi informativi OsMed con quelli dei Sistemi di Monitoraggio delle prescrizioni farmaceutiche. Questi sistemi, alcuni dei quali attivati a livello regionale, altri a livello di singole ASL, permettono di ricavare informazioni utili relative all' assistenza farmaceutica erogata alla popolazione assistita: nel 2004 è stato possibile osservare la prevalenza d'uso dei farmaci in una popolazione di oltre 7 milioni di assistibili, distribuiti prevalentemente nell'area Centro-Nord del Paese. Questo campione, sebbene non completamente rappresentativo in senso statistico, consente tuttavia di analizzare e comprendere quale peso abbiano alcuni principali fattori che determinano l'uso dei farmaci.

Alcune analisi circa l'appropriatezza d'uso dei medicinali nella medicina del territorio sono state effettuate grazie alla collaborazione della Società Italiana di Medicina Generale (SIMG), che ha messo a disposizione i dati raccolti da una propria rete informatica che collega oltre 500 medici di Medicina Generale.

Infine, la disponibilità di una serie storica di dati su base mensile, risalente ormai al 2000, ha consentito l'analisi delle principali componenti della dinamica temporale delle prescrizioni, a livello sia di gruppi terapeutici sia di singole molecole.

La spesa farmaceutica territoriale complessiva, pubblica e privata, del 2004 è stata di 19.185 milioni di euro ( $+5,5 \%$ rispetto al 2003) e ha fatto registrare un incremento del $9 \%$ della quota a carico del SSN e una contrazione del 2,6\% a carico del privato. Il SSN ha coperto oltre il $70 \%$ della spesa farmaceutica complessiva.

L'aumento fatto registrare dalla spesa farmaceutica pubblica è imputabile quasi interamente ad un aumento delle quantità prescritte 
$(+9,8 \%)$ e, in misura minore, allo spostamento della prescrizione verso farmaci più costosi (effetto mix $+0,6 \%)$. L'incremento è stato contenuto dalla diminuzione dei prezzi dell' $1 \%$.

La spesa per farmaci con brevetto scaduto (generici propriamente detti e generici branded o copie con nome di fantasia) ha rappresentato il $10,1 \%$ del mercato dei prodotti rimborsati dal SSN, facendo registrare un aumento dello $0,3 \%$ rispetto al 2003 .

La prescrizione di farmaci è stata più elevata nel Centro-Sud rispetto al Nord ed è risultata caratterizzata da una rilevante variabilità tra Regioni, con un range compreso le 659 DDD (Dosi Definite Die) del Trentino Alto Adige e le 947 DDD del Lazio. Lo sforamento del tetto programmato di spesa farmaceutica è stato indotto dagli elevati e più costosi consumi di poche Regioni del Centro-Sud.

Il rapporto evidenzia che nel corso del 2004 circa il $70 \%$ della popolazione italiana ha ricevuto almeno una prescrizione di farmaci. Il consumo e la spesa farmaceutica aumentano con l'invecchiamento: i soggetti con età superiore a 65 anni assorbono il 60\% dei volumi e dei valori della spesa farmaceutica; un assistibile con oltre 75 anni di età induce una spesa 11 volte superiore a quella di una persona di età compresa tra 25 e 34 anni.

Nel 2004 ogni abitante in Italia ha ricevuto in media 28 confezioni di farmaci, di cui 16 a carico del SSN e 12 acquistate privatamente. Le dosi di farmaco prescritte a carico del SSN sono state 784 ogni mille abitanti, con un aumento del $35 \%$ rispetto al 2000.

I farmaci del sistema cardiovascolare hanno assorbito da soli circa la metà delle dosi prescritte, con un aumento di oltre il $10 \%$ rispetto al 2003 e del $140 \%$ rispetto al 2000.

L'amlodipina, il ramipril e la nitroglicerina hanno fatto registrare consumi superiori alle 20 DDD ogni mille abitanti. Il farmaco a maggior consumo assoluto è stato ancora l'acido acetilsalicilico utilizzato come antiaggregante piastrinico, con 29 DDD ogni mille abitanti.

Al secondo posto in termini di consumi si trovano i farmaci del sistema gastrointestinale con oltre 1' $11 \%$ delle dosi. Rispetto al 2003 sono aumentati i consumi dei farmaci per l'apparato respiratorio e quelli per il sistema nervoso centrale.

Tra le categorie terapeutiche che hanno fatto segnare i maggiori incrementi di consumi e di spesa sono da citare le statine e gli omega 3, indicati nella terapia ipocolesterolemizzante, $\mathrm{i}$ bifosfonati per la prevenzione dell'osteoporosi e alcuni analgesici oppiacei utilizzati nella terapia del dolore.

Salvo qualche eccezione, i farmaci che hanno fatto registrare un maggior incremento di prescrizioni appartengono a classi terapeutiche utilizzate in patologie ad elevata prevalenza nella popolazione italiana, come l'ipertensione, le dislipidemie, le depressioni. È noto che queste patologie sono ancora oggi non diagnosticate in circa il $30-50 \%$ dei casi e che in almeno il 50\% dei soggetti diagnosticati la terapia non viene attuata o viene attuata in modo incompleto o inappropriato.

Soprattutto la spesa per farmaci cardiovascolari e per farmaci antidepressivi è destinata ancora a crescere nei prossimi anni, creando le condizioni per ulteriori sforamenti del tetto programmato di spesa farmaceutica.

Inoltre, altri campi terapeutici, come l'oncologia, saranno teatro di forti incrementi della spesa farmaceutica a causa dell'introduzione sul mercato di nuovi chemioterapici ad elevato costo.

Avere a disposizione un sistema di monitoraggio del consumo e della spesa farmaceutica è dunque essenziale per poter adottare tempestivamente i provvedimenti di controllo e di razionalizzazione necessari per continuare a mantenere sostenibile l'attuale avanzato sistema sanitario pubblico italiano. 